\title{
Care Networks of Home-dwelling Older Persons in the Netherlands: Proof of Concept of a Network Typology
}

\section{Wendy Kemper-Koebrugge ( $\sim$ wendy.kemper@han.nl )}

HAN University of Applied Sciences: Hogeschool van Arnhem en Nijmegen https://orcid.org/00000003-4468-9447

\section{Marian Adriaansen}

HAN University of Applied Sciences: Hogeschool van Arnhem en Nijmegen

\section{Miranda Laurant}

HAN University of Applied Sciences: Hogeschool van Arnhem en Nijmegen

\section{Michel Wensing}

Heidelberg University

\section{Research article}

Keywords: Older person, care network, informal care, qualitative methodologies, network type, network interaction

Posted Date: March 15th, 2021

DOI: https://doi.org/10.21203/rs.3.rs-310896/v1

License: (1) This work is licensed under a Creative Commons Attribution 4.0 International License. Read Full License 


\section{Abstract}

\section{Background}

Studies on care networks of home-dwelling older persons often focus on network constellation. However, looking at network interaction can be helpful to improve the support generated by the care network. A European study on diabetes patients found network types based on the interaction within a network, which can be beneficial (generative, proxy) or detrimental (struggling, avoidant) to support. This study explored whether these network types are present in care networks of home-dwelling older persons in the Netherlands and how these network types manifest in constellation or ways of interaction (negotiation, navigation and contagion).

\section{Methods}

The present study is a secondary analysis of a cross-sectional qualitative study of 16 care networks with home-dwelling older persons, informal caregivers and formal care providers conducted between March and September 2016. First, navigation, negotiation and contagion were analysed for each network; then the network type was determined for each network.

\section{Results}

We found nine proxy networks, four generative networks, two avoidant networks and one struggling network. In the proxy networks, all negotiation and navigation were centralised by the proxy. In generative networks, negotiation was possible if the older person could be reciprocal and the network supported this. In avoidant networks, informal and formal caregivers had to deal with an older person who refused support. In the struggling network, the underlying problem could not be addressed. Furthermore, two networks seemed to show a transition process from generative to proxy network.

\section{Conclusion}

We concluded that the network typology on interaction offered extra information about the functioning of the care network. It seems that the network type can change over time, but more research is needed to confirm this. This study suggests that interventions in avoidant or struggling networks are difficult. Also actions were aimed at developing proxy networks. Interventions maintaining or developing generative networks seem underused.

\section{Background}

With ageing populations and spending cuts in public healthcare, western countries are increasingly focusing on ageing at home with support from a network that is comprised of the older person's social network with or without formal care providers (1). Networks exhibit diversity in their constellations and the support they generate (2-4). 
Many studies in the last decades have focused on the constellation of these networks (3,5-11). These studies clarify structures of support and care, which emerge from interaction and act as context for interaction $(5,12)$. Recognition of different network constellations makes it possible to identify strengths and weaknesses in informal support and to see which network types are more likely to request formal help than others $(5,11,13)$.

Some studies have considered the nature and qualities of the relationships in a network, mostly aimed at the relationship of the older person with other network participants (14-16). Looking at the patterns of interaction in the total network relationships could be helpful to improve the support generated by the network. A study on care networks of patients with type 2 diabetes developed a network typology for network interaction (17). That study looked at all relationships in the network through three predetermined network mechanisms: navigation to sources of support, negotiation of support, and contagion of supportive behaviours. This led to distinguish four network types: 1) Generative networks: diverse and beneficial to the individual because many actors provide and seek support; 2) Proxy networks: where coordination is delegated to one super-helper. This network could be beneficial, but it is often frail, particularly when it relies on an informal caregiver with few other connections; 3) Avoidant networks: where support is not negotiated; and 4) Struggling networks: where managing the chronic condition is a struggle or not prioritised by participants in the care network.

This network typology was derived from research involving patients with type 2 diabetes in six European countries (17). $80 \%$ of patients in the sample were younger than 74 years, $41 \%$ had no other diseases, and $65 \%$ rated their general health as very good or good. It is unknown whether this network typology can also be used to explore network interaction in the care networks of home-dwelling older persons 75 years and over, who often have multi-morbidity, and are facing a decline in health as they age further.

A network typology can help professionals understand patterns in network interactions and how the network can be influenced. We assumed that this network typology would be helpful for developing interventions that formal care providers can use to improve the functioning of older persons' care networks.

This led to the present study, which explored the generalization of the typology on the basis of the following research questions:

- Are the network types described by Kennedy et al. (2014), which relate to patterns of interactions, present in care networks of home-dwelling older persons in the Netherlands?

How does the network type manifest itself in constellation, and/or in interaction (negotiation, navigation or contagion) in these care networks?

\section{Methods}


We performed a qualitative interview study (Blinded for review \#2) and performed (cross sectional) data analysis to explore network interaction. We interviewed three parties per care network separately: the older person, an informal caregiver and a formal care provider. The research team had a background in nursing, education and social science and had no prior relation to the respondents. The design and report of the study followed the COREQ guidelines (18)

\section{Ethical Approval}

Permission was obtained from the practice-oriented research ethical advisory board Blinded for review \#1, Nijmegen, the Netherlands, ACPO 24.03/16.

\section{Study Sample and Data Collection}

The study sampled care networks that provide informal and formal care to an older person aged 75 years or older who has multiple chronic illnesses (Blinded for review \#2). The older person chose the informal caregiver and formal care provider to study. We recruited respondents via communities and organisations to get a purposeful sample: a church community, home care organisations, a local welfare organisation, home care services, all in the eastern part of the Netherlands. The principal researcher (Blinded for review \#3) first communicated with contact people from organisations who then selected and approached clients personally. They provided those clients more information about the study and its goals.

At the start of the interview, respondents signed an informed consent form. Respondents are quoted in this article by network number $(N)$ and network party: the older person $(A)$, the informal caregiver $(B)$ and the formal care provider (C). Data was collected between March and September 2016. Interviews with participants were conducted no more than two weeks apart to avoid having differences in the network situation blur the differences in perceptions of network interaction. Interviews were held in the respondent's home or at the office. No other people were present besides the respondent and researcher. No repeat interviews were carried out.

\section{Interviews}

The semi-structured interviews followed an interview guide with open-ended questions (Blinded for review \#2). The interview guide was identical for all parties, apart from formulating the question from the perspective of the network party. Respondents reflected on the current situation in the network from their own perspective.

The questions about network interaction started by exploring the network constellation: which informal caregivers and formal care providers offer support. Next, we explored ways of navigation, negotiation and contagion by asking open questions and requesting specific examples of situations. Every network mechanism had leading questions: 
- Navigation: 'If something changes in the situation, how would Mrs/Mr address this? How do you look for people or organisations that could help?'

- Negotiation: 'How do participants interact to find out what the older person needs and to divide care tasks?'

- Contagion: 'How would you define positive or negatives influences in or around the network? How is this influence strengthened by the way people interact?'

Blinded for review\#3 was the primary researcher and Blinded for review \#3, JN, LB and MP conducted interviews and attended training beforehand. The interviews lasted about one hour. The researchers recorded the interviews and transcribed them verbatim. The researchers discussed data saturation in the research team.

\section{Data Analysis}

First, two researchers (Blinded for review\#3 and JN) coded the data separately in ATLAS-ti, a software program for qualitative analysis, then compared and discussed them. Disagreements were solved in a consensus meeting with a third researcher (Blinded for review \#4). Second, they analysed meaningful units on constellation, navigation, negotiation and contagion per network. Third, using these units, the researchers scored which criteria of the network typology applied to each network [see Table 1]:

- $1=$ respondents clearly mentioned this criterion or an example illustrated this criterion and stories among respondents were consistent

- $1 / 2=$ this criterion could be derived from the respondents' stories but stories conflicted between respondents

- $0=$ the criterion was not supported by the respondents' stories

A score only was applied when the criterion fit the current situation in the care network. Based on the scores, the researchers applied the network type which scored highest related to the maximum criteria of that network type

[Insert] Table 1: Criteria in network typology and network constellation and interaction

\section{Results}

\section{Network Characteristics}

In total, 16 networks were included: 12 networks with all three network parties and 4 networks with two network parties. Three networks with only one respondent were excluded from this secondary analysis. Different situations caused networks to miss a network party: one older person did not name a formal care provider, one older person felt interviewing domestic help was too much of a burden on the domestic 
help, one informal caregiver was not reached, and one older person with dementia was unable to participate in the planned interview.

\section{Network Types}

Applying the criteria of the network typology (Table 1) led to the following result (Table 2).

[Insert] Table 2: Fitness of network typology

We found all the network types in our study population. Every network in the sample could be assigned to a network type, meaning that they only scored on that network type (14 out of 16 networks) or scored high on that network type. Two networks (N12 and N13) scored on two network types, with one network type scoring higher than the other. This meant we applied the network type of which most criteria were met.

The proxy network was the most present (9 times), followed by the generative network (4 times), the avoidant network (2 times) and the struggling network (1 time).

Participants had different views on the interactions in the network. If we would have spoken only to the older person in N10, for example, the network type would have been avoidant, but through other stories of network participants the proxy became clear:

'We had two multidisciplinary meetings about him this year because we were worried. He doesn't find that all that impressive. He thinks it is unimportant to shower more than once every two weeks. Then you face an ethical dilemma: should you let him or not?'(N10C)

Stories from informal and formal care often offered extra information. Asking the older person 'who or what supports you' clarified what part of the care network that person noticed and what attitude they adopted towards support provided.

With formal care providers, it made a difference if they comprehended the older person's situation outside their own area of responsibility. For example, a social worker who supported the older person in day-care activities had no knowledge of the situation at home.

\section{Proxy networks.}

Quotes that led to applying criteria of this network type were all descriptions of a proxy negotiating or navigating. Two types of proxy were found: an overall proxy (one proxy negotiated the informal and formal care) and a split proxy network (the informal and formal care each had their own proxy). The overall proxy is most clearly illustrated in N3 and N4, which was also visible in the views on network constellation. The proxy in N3 and N4 organized other networks. The older person named few network participants. Only through the proxy's stories the richness of the care network became visible. The informal caregiver performed all negotiation and navigation: 
'He accepts it very well, he says; my wife can't let it go. She actually works with him the entire 24 hours a day. I am in contact with his wife in particular. With some clients, you are the proxy in a network as a nurse, but here the partner does that and she really wants to do it herself.' (N4C)

The older person in N3 described the value of contacts with acquaintances, but they are all organized by the proxy. Formal care in N3 explains that if the proxy did not organize this, the older person would come nowhere.

In split proxy networks, there was limited contact between the proxies in informal and formal care. The informal proxy negotiated informal care, while the formal proxy navigated to other formal care. Sometimes, an informal proxy was installed to stabilise family relations.

In N1, the stories of the informal and formal care showed that they tried to come and stay in contact, but they felt the older person was not really interested in them or the interaction felt negative:

'70-80\% of the initiatives comes from us; if I indicate it, she will take care of it. That goes through us or through that daughter' (N1C).

N9 and N10 showed that a proxy network is not always an older person resigning control to the network; sometimes it involves informal or formal care taking control. They described their ways of dealing with this older person:

'If I do too much at one time, he holds back. He has to come to trust the volunteer or he will send them away' (N9B).

\section{Generative networks.}

Descriptions of reciprocity and references to a community or living environment led us to apply criteria of this network type. In the networks that only had criteria from this network type, the older persons felt physically capable of doing something for other people: for example babysitting grandchildren. The diversity in informal care named in the network constellation was larger in the generative networks than in the other network types, with acquaintances named (e.g. people from the neighbourhood or church community). Especially the church community meant the older persons had more available support without having to maintain personal relationships with every person. Informal caregivers often cared for more older persons in the church community and named values as reason (contagion), as confirmed by the practice nurse from the general practitioner:

'I know the community in which the older person lives, I know who I'm dealing with, which implies I have to monitor less' (N14C).

In most generative networks, the position of the older person reflected their past role in the community. However, in N17, the older person negotiated her own support and was able to maintain a generative 
network in a different way:

'She can be manipulative. The general practitioner even sometimes admits he does something for her that he intentionally did not want to do' (N17C).

\section{Struggling networks.}

N8 was a struggling network with the criterion prioritising other problems most clearly present. The older person had a mentally disabled son who officially lived elsewhere but was often present in the household and demanded attention. Negotiation was difficult. The son's and the older persons formal care network were not in contact with each other due to privacy regulations, and the older person's formal care provider felt that care for the older person could not be optimised. Also, the older person's informal caregiver distanced herself from the situation because she fought with her brother.

\section{Avoidant networks.}

Avoidance of support and lack of discussion or denial defined the avoidant networks. The older person felt in control and the informal and formal caregivers stated that the older person refused support. They related that refusal to character:

'If you look in the news at people with loneliness, then he is not a representative of lonely people because he chooses to do this himself' (N9B).

The informal and formal caregivers had the dilemma to refrain from engaging with the situation or to take control when situations became unsafe:

'I am sometimes dictatorial: refusing what is not safe' (N9B).

\section{Can the network type change?}

'I am not such a fan of other humans, let's face it.' (N6A)

'But at some point, you just keep asking. Do not apply too much pressure. Because often she doesn't want it anymore. Until she says, well, I'm going to have a look.' (N6C)

In N2 the informal caregiver worried about the future, in which her effort to maintain the proxy network could change into a struggling network. Managing the support for her father with dementia took priority over the support for her mother with paralysis. At the time of the research the situation was stable but if the condition of her father changed, the network could become a struggling network in which a balance would have to be found between supporting her father and her mother. The informal proxy claimed to be aware of this situation: 
'I know there is a bomb lying there. I just hope my father dies before we have to bring him to a nursing home, so afterwards we can do something for my mother.' (N2B)

Furthermore, network N12 and N13 seemed to show a transition process from generative towards a proxy network. In those networks, parties attributed this change to the older person's deteriorating health. N12 scored 3 out of 4 on proxy and 4 out of 4 on generative, N13 the other way around. The generative surroundings kept $\mathrm{N} 12$ generative because the contagion in the church community was to include all older persons in community activities. The older person in N13 did not use the generative possibilities, so the informal caregiver turned to a proxy network:

'She has potential support in the surroundings, but she has trouble accepting what she cannot do anymore, which prevents her from using care and accepting emotional support.' (N13B)

\section{Discussion}

Determining the network type based on interaction proved possible in care networks of home-dwelling older persons. The network type manifested in the respondents' narratives and their stories were complementary. Further, in contrast to portraying networks in constellation, asking participants about how they negotiate, navigate in the network and contagion of supportive behaviour, gave insight into underlying patterns and reasons for the network's configuration and interaction patterns.

Network strategies aim at individual behavioural change, such as enlarging the network and changing the network configuration (19). Network interaction adds another perspective: that interaction can be positive (generative/proxy) or negative (struggling/avoiding), which is associated with the amount of social support. Insight into interaction patterns showed why it is difficult to enlarge a network. Nevertheless, network types may change depending on the older person's health or the wishes and needs of other network parties (11).

This study suggested that interventions aimed at increasing support are difficult in avoidant or struggling networks. In a struggling network, effective negotiation and navigation are overshadowed by other problems. This means that participants have to address underlying problems before other network interaction can develop. Informal and formal care in an avoidant network should first focus on gaining trust and creating bonds with people in the community in small steps, so the older person dares to ask for help (20). With the exception of generative networks, contagion of supportive behaviours was not mentioned. Contagion appears to be a difficult concept to recognize.

The proxy network was the most common type in this study. A proxy can fill holes in the network left by age-related changes and life events $(3,21)$. Creating a proxy in informal or formal care is an intervention applied by informal and formal care to organise contact and distance themselves from interaction in the network of informal caregivers (2). However, the proxy network is vulnerable because changes in the network are dependent on this often informal proxy. Caregiver distress is a significant predictor of nursing 
home placement (22). Creating more than one proxy could be a helpful intervention to divide support tasks more broadly and therewith spread the risks of overloading one informal caregiver.

In the sample, we saw the struggle to maintain a generative network. Networks undergo age-related changes in which older persons lose network ties because of life events and lose non-kin relationships when they become more selective $(3,11)$. Participants were not focused on maintaining a generative network or exploring possibilities for reciprocity from the older person, even though shaping reciprocal relationships is crucial to give an older person a feeling of independence and a foundation for a caring relationship $(23,24)$. Also weaker ties appear more durable and less vulnerable to loss over time than stronger ties (25).

This study had some limitations. We interviewed only three participants per network and, in some cases, only two participants. We may have missed present generative networks, but with three respondents we gain insight in the interaction between the most involved participants. There could be bias in the types of informal and formal care respondents because the older persons chose respondents.

In addition, network types were not divided proportionally. A selection bias in participation of struggling networks seems logical. The strong prevalence of proxy networks could be caused by the presence of formal care providers in these care networks, but also could be the way they preferred to organise the network. This requires more research.

Since proxy networks in which the informal caregiver is the proxy are often overburdened, there is a need for more research about how to split proxies and spread responsibilities among informal and formal caregivers. Research on possibilities to maintain generative networks or create access to generative networks in the neighbourhood could give informal caregivers and formal care providers a different range of interventions to organise the support for the older person.

Another limitation of our study was the cross-sectional design. We interviewed respondents once. Following care networks over time and deepening the knowledge of transition processes in network types could help define interventions that help care networks become positive in their interactions and generate support.

We conclude that the network types described by Kennedy et al. (2014) were present in the care networks of home-dwelling older persons in the Netherlands. In proxy networks, negotiation and navigation was centralised by the proxy. In generative networks, negotiation and navigation was possible because the older person was reciprocal and network surroundings were supportive. Only in generative networks we found examples of contagion. In avoidant networks, mostly caused by older person's avoidant behaviour, negotiation and navigation was limited. In struggling networks, underlying problems could not be addressed, which hampered effective negotiation or navigation.

Interaction gave more information than constellation about the functioning of the care network. In the constellation participants can be named, but that does not imply they are taking part in interaction. 
Formal care providers should include knowledge of network types into their strategies to strengthen the care network.

\section{Abbreviations}

Not applicable

\section{Declarations}

\section{Ethical Approval and consent to participate}

Permission was obtained from the practice-oriented research ethical advisory board in the Faculty of Health, Behaviour and Society at HAN University of Applied Sciences, ACPO 24.03/16.

Written informed consent was obtained from all participants.

\section{Consent for publication}

not applicable

\section{Availability of data and materials}

The datasets used and analysed during the current study are available from the corresponding author on reasonable request

\section{Competing Interests}

The authors declare that they have no competing interests. The authors alone are responsible for the content and writing of the paper.

\section{Funding}

This research received no specific grant from any funding agency in the public, commercial, or not-forprofit sectors.

\section{Author Contribution}

WK planned the study, collected and analysed data and wrote the paper. MA supervised the interpretation of findings and contributed to writing the manuscript. ML supervised the study, data collection, analysis and interpretation of findings and contributed to writing the manuscript. MW supervised the study design and interpretation of findings and contributed to writing the manuscript. All agreed with the final manuscript. 


\section{Acknowledgements}

I thank Joke Nijenhuis-van Weert (JN) for assistance with the interviews and help with the analysis, which greatly improved the study results. I also thank Lisanne Boer (LB) and Moniek Peeters (MP) for assistance with the interviews.

\section{References}

(1) Verver, D., Merten, H., Wagner, C., \& Robben, P. (2018). Care and support for older persons in the netherlands living independently (HEALTH \& SOCIAL CARE IN THE COMMUNITY; MAY 2018, 26 3, pe404pe414, 11p ed.) doi:https://doi.org/10.1111/hsc.12539

(2) Kemper-Koebrugge W, Adriaansen M, Laurant M, Wensing M. Actions to influence the care network of homedwelling elderly people: a qualitative study. Health and Social Care in the Community 2019, 27(4), 973-981. doi.org/10.1111/hsc.12714.

(3) Ellwardt L, Aartsen M, van Tilburg T. Types of Non-kin Networks and Their Association With Survival in Late Adulthood: A Latent Class Approach. JOURNALS OF GERONTOLOGY SERIES B-PSYCHOLOGICAL SCIENCES AND SOCIAL SCIENCES 2017;72(4):694-705, doi:https://doi.org/10.1093/geronb/gbw142.

(4) Holt-Lunstad J, Smith TB, Layton JB. Social Relationships and Mortality Risk: A Meta-analytic Review. PLoS Medicine 2010 07;7(7):1-20, doi:10.1371/journal.pmed.1000316.

(5) Wenger GC. A network typology: From theory to practice. Journal of Aging Studies 1991 01/01;5(2):147-162.

(6) Fiori KL, Antonucci TC, Cortina KS. Social Network Typologies and Mental Health Among Older persons. J Gerontol B Psychol Sci Soc Sci 2006 01;61(1):25-32.

(7) Li T, Zhang Y. Social network types and the health of older persons: Exploring reciprocal associations. Soc Sci Med 2015;130:59-68, doi:10.1016/j.socscimed.2015.02.007.

(8) Litwin H, Shiovitz-Ezra S. The Association of Background and Network Type Among Older Americans: Is "Who You Are" Related to "Who You Are With"? Res Aging 2011;33(6):735-759.

(9) Wojszel ZB, Politynska B. The structure and functional correlates of social support networks of people in advanced old age living in chosen urban and rural areas in poland: A cross-sectional study. Eur $\mathrm{J}$ Ageing 2020 10/16, doi:10.1007/s10433-020-00583-6.

(10) Rogero-Garcia J, Rosenberg MW. Paid and unpaid support received by co-resident informal caregivers attending to community-dwelling older persons in Spain. European Journal of Ageing 2011(2):95. 
(11) Litwin H, Levinsky M, Schwartz E. Network type, transition patterns and well-being among older Europeans. European Journal of Ageing 2020(2):241, doi:10.1007/s10433-019-00545-7.

(12) Vassilev I, Rogers A, Kennedy A, Wensing M, Koetsenruijter J, Orlando R, et al. Social Network Type and Long-Term Condition Management Support: A Cross-Sectional Study in Six European Countries. PLoS ONE 2016 08/18;11(8):1-15, doi:10.1371/journal.pone.0161027

(13) Wenger GC, Tucker I. Using network variation in practice: identification of support network type. Health \& Social Care in the Community 2002 01;10(1):28-35, doi:10.1046/j.0966-0410.2001.00339.

(14) Jacobs MT, van Groenou, Marjolein I. Broese, Aartsen MJ, Deeg DJH. Diversity in Older persons' Care Networks: The Added Value of Individual Beliefs and Social Network Proximity. JOURNALS OF GERONTOLOGY SERIES B-PSYCHOLOGICAL SCIENCES AND SOCIAL SCIENCES 2018;73(2):326-336, doi:https://doi.org/10.1093/geronb/gbw012.

(15) Nguyen AW. Variations in Social Network Type Membership Among Older African Americans, Caribbean Blacks, and Non-Hispanic Whites. JOURNALS OF GERONTOLOGY SERIES B-PSYCHOLOGICAL SCIENCES AND SOCIAL SCIENCES 2017;72(4):716-726.

(16) Vos W, Boekel vH, Janssen L, Leenders M, Luijkx K. Exploring the impact of social network change: Experiences of older persons ageing in place. Health \& Social Care in the Community 2020;28(1):116-126, doi:10.1111/hsc. 12846.

(17) Kennedy A, Rogers A, Vassilev I, Todorova E, Roukova P, Foss C, et al. Dynamics and nature of support in the personal networks of people with type 2 diabetes living in Europe: qualitative analysis of network properties. Health Expectations, 2015, (6): 3172. https://doi.org/10.1111/hex.12306.

(18) Tong A, Sainsbury P, Craig J. Consolidated criteria for reporting qualitative research (COREQ): a 32item checklist for interviews and focus groups. . International Journal for Quality in Health Care: Journal of the International Society for Quality in Health Care 2007, 19(6), 349-357.

(19) Spencer-Bonilla G, Ponce OJ, Rodriguez-Gutierrez R, Alvarez-Villalobos N, Larrea-Mantilla L, Montori $\mathrm{VM}$, et al. A systematic review and meta-analysis of trials of social network interventions in type 2 diabetes. BMJ OPEN 2017;7(8).

(20) Mizuochi M. Social capital and refraining from medical care among elderly people in Japan. BMC Health Services Research 2016 08/02;16:1-9, doi:10.1186/s12913-016-1599-8.

(21) Wenger GC. A network typology: From theory to practice. Journal of Aging Studies 1991;5:147-162.

(22) Maxwell CJ, Campitelli MA, Diong C, Mondor L, Hogan DB, Amuah JE, et al. Variation in the health outcomes associated with frailty among home care clients: relevance of caregiver distress and client sex. BMC GERIATR 2018 09/12;18(1):1-11, doi:10.1186/s12877-018-0899-8 
(23) Lewinter M. Reciprocities in caregiving relationships in Danish elder care. Journal of Aging Studies 2003 08;17(3):357, doi:10.1016/S0890-4065(03)00025-2.

(24) Fyrand L. Reciprocity: A Predictor of Mental Health and Continuity in Elderly People's Relationships? A Review. Current Gerontology \& Geratrics Research 2010 01:1 Volume 2010, Article ID 340161 ed.) doi:doi:10.1155/2010/340161.

(25) Rogers A, Brooks H, Vassilev I, Anne K, Christian B, David R. Why less may be more: a mixed methods study of the work and relatedness of 'weak ties' in supporting long-term condition self-management. Implementation Science 2014 02;9(1):1-22, doi:10.1186/1748-5908-9-19.

\section{Tables}

Table 1: Criteria in network typology and network constellation and interaction 


\begin{tabular}{|c|c|c|c|}
\hline & $\begin{array}{l}\text { Criteria of network type } \\
\text { (Kennedy et al., 2014) }\end{array}$ & $\begin{array}{l}\text { Manifestation in } \\
\text { network } \\
\text { constellation }\end{array}$ & $\begin{array}{l}\text { Manifestation in network } \\
\text { interaction (navigation, negotiation, } \\
\text { contagion) }\end{array}$ \\
\hline \multirow[t]{4}{*}{$\begin{array}{l}\text { Generative } \\
\text { network }\end{array}$} & $\begin{array}{l}\text { 1A Many actors, diverse } \\
\text { participants }\end{array}$ & $\begin{array}{l}\text { Family, } \\
\text { organisations, } \\
\text { more friends, } \\
\text { neighbours and } \\
\text { groups } \\
\text { mentioned }\end{array}$ & \\
\hline & $\begin{array}{l}\text { 1B Active weak-tie } \\
\text { connections }\end{array}$ & $\begin{array}{l}\text { Older person is } \\
\text { part of groups } \\
\text { and has contact } \\
\text { with (loose) } \\
\text { acquaintances }\end{array}$ & $\begin{array}{l}\text { Groups and acquaintances present, } \\
\text { Contact in networked groups, } \\
\text { references to values in these groups } \\
\text { (contagion) }\end{array}$ \\
\hline & $\begin{array}{l}\text { 1C Participants make } \\
\text { healthy changes } \\
\text { sustainable }\end{array}$ & & $\begin{array}{l}\text { Role network participants in } \\
\text { change, group exercises } \\
\text { (contagion) }\end{array}$ \\
\hline & $\begin{array}{l}\text { 1D Participants are able to } \\
\text { reciprocate }\end{array}$ & & $\begin{array}{l}\text { Reciprocity, participation older } \\
\text { person in groups }\end{array}$ \\
\hline \multirow[t]{4}{*}{$\begin{array}{l}\text { Proxy } \\
\text { network }\end{array}$} & $\begin{array}{l}\text { 2A Network managed by } \\
\text { others on behalf of the } \\
\text { older person, }\end{array}$ & $\begin{array}{l}\text { Mostly family } \\
\text { names, formal } \\
\text { care }\end{array}$ & $\begin{array}{l}\text { References to a proxy, } \\
\text { Negotiation often family-centred or } \\
\text { focused on health professionals, } \\
\text { Limited contagion from formal care }\end{array}$ \\
\hline & 2B Close bond connections & $\begin{array}{l}\text { Classical strong- } \\
\text { tie relationships: } \\
\text { spouse, child, } \\
\text { partner, sibling }\end{array}$ & Mostly daily, intimate contact, \\
\hline & $\begin{array}{l}2 \mathrm{C} \text { Changes are dependent } \\
\text { on a close super-helper }\end{array}$ & & $\begin{array}{l}\text { Negotiation: References to } \\
\text { coordination of single super-helper, } \\
\text { change in care network via super- } \\
\text { helper }\end{array}$ \\
\hline & $\begin{array}{l}\text { 2D Trust that care was } \\
\text { being actively managed } \\
\text { and discussed with little } \\
\text { need for the older person to } \\
\text { be in control. }\end{array}$ & & $\begin{array}{l}\text { Older person describes trust in and } \\
\text { often dependency on super-helper. } \\
\text { Delegate control to their network }\end{array}$ \\
\hline $\begin{array}{l}\text { Avoidant } \\
\text { network }\end{array}$ & $\begin{array}{l}\text { 3A Older person avoids } \\
\text { help and support }\end{array}$ & $\begin{array}{l}\text { Small number of } \\
\text { participants }\end{array}$ & $\begin{array}{l}\text { References informal and formal } \\
\text { care on avoidance by older person, } \\
\text { No navigation by older person, } \\
\text { mentions of navigation without } \\
\text { older person }\end{array}$ \\
\hline & $\begin{array}{l}\text { 3B Denial and lack of } \\
\text { discussion in the network }\end{array}$ & & $\begin{array}{l}\text { No mention of negotiation. Control } \\
\text { older person, no contagion }\end{array}$ \\
\hline
\end{tabular}




\begin{tabular}{|c|c|c|c|}
\hline & $\begin{array}{l}\text { Criteria of network type } \\
\text { (Kennedy et al., 2014) }\end{array}$ & $\begin{array}{l}\text { Manifestation in } \\
\text { network } \\
\text { constellation }\end{array}$ & $\begin{array}{l}\text { Manifestation in network } \\
\text { interaction (navigation, negotiation, } \\
\text { contagion) }\end{array}$ \\
\hline & \multicolumn{3}{|l|}{$\begin{array}{l}\text { 3C Need for hypercontrol } \\
\text { (disease) }\end{array}$} \\
\hline \multirow[t]{4}{*}{$\begin{array}{l}\text { Struggling } \\
\text { network }\end{array}$} & \multirow[t]{2}{*}{$\begin{array}{l}\text { 4A Hidden nature of the } \\
\text { chronic conditions }\end{array}$} & & $\begin{array}{l}\text { Extreme different views on } \\
\text { situation, }\end{array}$ \\
\hline & & & $\begin{array}{l}\text { network does not see the condition, } \\
\text { limited/poorly understood } \\
\text { examples of health behaviours }\end{array}$ \\
\hline & $\begin{array}{l}\text { 4B Prioritising other } \\
\text { problems }\end{array}$ & & $\begin{array}{l}\text { References to worries and } \\
\text { consequences, financial } \\
\text { worries/other health conditions/ } \\
\text { complicated family life, Influences } \\
\text { of worries on care network }\end{array}$ \\
\hline & $\begin{array}{l}\text { 4C Some cases: confused } \\
\text { by unhelpful advice }\end{array}$ & & Mention unhelpful advice \\
\hline
\end{tabular}

Table 2: Fitness of network typology

\begin{tabular}{|llllll|}
\hline & $\begin{array}{l}\text { Avoidant } \\
\text { network }\end{array}$ & $\begin{array}{l}\text { Struggling } \\
\text { network }\end{array}$ & $\begin{array}{l}\text { Generative } \\
\text { network }\end{array}$ & Proxy network & Total \\
$\begin{array}{l}\text { Number of } \\
\text { networks }\end{array}$ & 2 & 1 & 4 & 9 & 16 \\
\hline $\begin{array}{l}\text { Score on } \\
\text { network type }\end{array}$ & $\begin{array}{l}\text { 3 out of 3 (1 } \\
\text { network) }\end{array}$ & $\begin{array}{l}\text { 3 out of 3 } \\
\text { 2 out of 3 (1 } \\
\text { network) }\end{array}$ & $\begin{array}{l}\text { 4 out of 4 (3 network) } \\
\text { networks) }\end{array}$ & $\begin{array}{l}\text { 4 out of 4 (7 } \\
\text { networks) } \\
\text { 3,5 out of 4 (1 } \\
\text { network) }\end{array}$ & $\begin{array}{l}\text { 2,5 out of 4 (2 } \\
\text { networks) }\end{array}$ \\
\hline
\end{tabular}

\section{Supplementary Files}

This is a list of supplementary files associated with this preprint. Click to download.

- COREQCheckliststudynetworktype.docx 